\title{
On a fractional step-splitting scheme for the Cahn-Hilliard equation
}

\author{
AA Aderogba, M Chapwanya, and JK Djoko \\ Department of Mathematics \& Applied Mathematics, University of Pretoria, Pretoria 0002, South Africa
}

\begin{abstract}
For a partial differential equation with a fourth order derivative such as the CahnHilliard equation, it is always a challenge to design numerical schemes that can handle the restrictive time step introduced by this higher order term. In this work, a fractional splitting method is employed to isolate the convective, the nonlinear second order and the fourth order differential terms. The full equation is then solved by consistent schemes for each differential term independently. In addition to validating the second order accuracy, we will demonstrate the efficiency of the proposed method by validating the dissipation of the Ginzburg-Landau energy and the coarsening properties of the solution.
\end{abstract}

Keyword: Cahn-Hilliard equation; hyperbolic; fractional step-split; numerical solution. PACS: 65M06; 65M08; 97N40

\section{Introduction}

In this work, we consider a finite difference approximation of the one dimensional convective Cahn-Hilliard $(\mathrm{cCH})$ equation

$$
u_{t}+\gamma^{2} u_{x x x x}=\phi(u)_{x x}+\delta u u_{x}, \quad x \in \Omega, 0<t,
$$

where $\Omega \in(a, L)$ and

$$
\phi(u)=\alpha u^{3}-u, \quad \alpha>0 .
$$

This equation is a successful model of phase transition observed in alloys, glasses, polymer solution and binary liquid mixtures (see for example Elliott and French (1987); Elliott and Songmu (1986); Novick-Cohen and Segel (1984), and the literatures therein). As a model for phase transition, $u$ is the perturbation of the concentration of one of the phases (Golovin et al., 2001; Watson, 2003); $\gamma$ and $\delta>0$ are dimensionless parameters. When the driving force, $\delta \rightarrow 0$ we recover the traditional Cahn-Hilliard $(\mathrm{CH})$ equation (Elliott and French,

\footnotetext{
*Corresponding author. m.chapwanya@up.ac.za
} 
1987), and for large $\delta$, the equation behaves as the Kuramoto-Sivashinsky equation, (Golovin et al., 2001).

The $\mathrm{cCH}$ equation has attracted the attention of many researchers in recent years. Emmott and Bray (1996) observed that the driving force $\delta$ has an asymmetric effect on the solution for a kink when $\gamma=\alpha=1$. They also noted that the dynamics of a kink-antikink pair is a function of $\delta^{-1}$ and the length of the interface. Later, Golovin et al. (2001) noted that this kink-antikink pair occurs as a final result of coarsening on a periodic domain. This was confirmed by the work of Watson (2003) and Watson et al. (2003). Watson (2003) derived the $\mathrm{cCH}$ equation for the dynamics of crystal growth and performed his simulation on a periodic domain with random initial data. For various values of the driving force, $0<\delta \leq 1$, his results agree with earlier observations in Golovin et al. (2001) on the kink-antikink pair. Recently, Podolyn et al. (2005) investigated the dynamics of domain walls (kinks) governed by the $\mathrm{cCH}$ equation with special interest in the dynamics of kink pairs and triplets that play a crucial role in the coarsening process. They derived an analytical formula that describes the motion of the kink pairs and the triplets.

For $\delta=0$, equation (1.1) reduces to

$$
u_{t}+\gamma^{2} u_{x x x x}=\phi(u)_{x x}, \quad x \in \Omega, 0<t,
$$

which is an equation of conservation of mass with flux

$$
J=-\left[\phi(u)-\gamma^{2} u_{x x}\right]_{x} .
$$

It has been observed that the critical points of the Ginzburg-Landau free energy form

$$
\mathcal{E}=\int_{0}^{L}\left(F(u)+\frac{\gamma^{2}}{2} u_{x}^{2}\right) d x
$$

where $F$ is a Lyapunov functional,

$$
F(u)=\int_{0}^{u} \phi(s) d s
$$

is a steady state solution of the CH equation (Elliott and Songmu, 1986; Novick-Cohen and Segel, 1984) given the boundary conditions

$$
u_{x}=\gamma^{2} u_{x x x}-\phi(u)_{x}=0, \quad x \in \partial \Omega .
$$

More theoretical results can be found here (Elliott and Songmu, 1986; Novick-Cohen and Segel, 1984). In the work of Du and Nicolaides (1991), a Dirichlet problem of the CH equation (1.2) was formulated. They proved the existence and uniqueness of the solution based on the compactness method of a semi-discrete approximation sequence and a new finite element based fully discrete scheme with a Lyapunov functional. The Lyapunov functional helped them to obtain point wise estimates of the solution. Most of these theoretical results were validated computationally by several authors (see De Mello and Silveira Filho (2005); Eyre (1998); Lee et al. (2011) among others).

The numerical solution of entire $\mathrm{cCH}$ or $\mathrm{CH}$ equation have been investigated by various researchers using finite difference (Christlieb et al., 2012; Cueto-Felgueroso and Peraire, 2008; 
Du and Nicolaides, 1991; Eyre, 1998), finite elements (Elliott and French, 1987, 1989; Wells et al., 2006), boundary integral (Dehghan and Mirzaei, 2009), Fourier spectral (Zhu et al., 1999) methods - to name a few. In this work we consider finite difference schemes for both the $\mathrm{cCH}$ and $\mathrm{CH}$ equations. Among the most common explicit schemes is the Euler scheme which requires a restrictive $\Delta t$ of the order of $\gamma^{2}(\Delta x)^{4}$. On the other hand, implicit schemes will require that a system of nonlinear algebraic equations be solved - with a possible choice being the Newton method, which is again costly in terms of computer time. Shen and Yang (2010) investigated the stability of some numerical schemes for Allen-Cahn and the Cahn-Hilliard equations and observed that while implicit schemes were unconditionally stable; the semi-implicit schemes without stabilization are energy stable with reasonable condition and the first order case of the stabilized version is unconditionally energy stable. An unconditionally gradient method based on splitting of the contractive and the expansive terms across time steps was introduced by Eyre (1998). This same method was employed later by De Mello and Silveira Filho (2005) to solve the CH equation (1.2) in one, two and three dimensions based on a free boundary condition. Cueto-Felgueroso and Peraire (2008) employed a time adaptive procedure to solve a two dimensional $\mathrm{CH}$ problem. Elliott and French (1987) observed computationally that the solution will blow up in a finite time if the initial data is large and $\alpha<0$. The same authors (Elliott and French, 1989), later designed a semi-discrete finite element scheme and employed it to derive optimal error bounds in various norms.

Several properties of the $\mathrm{cCH}$ equation are documented in the literature. For example, in a model of crystal growth, Watson (2003) observed that the broken up-down symmetry is captured by the Burger's convection. Also, the matched asymptotic analysis performed by Watson et al. (2003) led to a kink/anti-kink sharp interface theory. It is revealed that there is an induction of a convective-diffusive flux of $u(x, t)$ due to the asymmetry between a kink and anti-kink which drives these discontinuities in a manner given by the Rankine-Hugoniot relation. These observations compared to the entire equation suggest three different components with special behavior: the convective term (known to introduce discontinuous solution in finite time); the nonlinear degenerate diffusion term (having a stabilizing effect) and the linear fourth order term (which possesses a stabilizing effect). Thus a true numerical solution has to preserve these properties of the full problem, hence here we treat each component separately.

The goal of this paper is to apply an operator split method to equation (1.1) thereby resolving the numerical difficulties outlined above which are associated with solving the complete equation in its entire form. Apart from the fact that the fractional step method substantially reduces computational time, it has the advantage of simplifying a complex process efficiently. This method permits the treatment of each segment of the original equation separately. Each of the subproblems is solved numerically using finite volume methods in such a way as to preserve the qualitative properties of the solutions related to each of the continuous subproblems. In particular, we split equation (1.1) using a fractional time step algorithm following the work of, for example, Otto and Giacomelli (2005); Yong-Jung et al. (2008). The stability of the method is guaranteed as long as the properties of each subproblem are preserved, see for example Heldge et al. (2010). We split equation (1.1) into three 
subproblems: the hyperbolic equation

$$
u_{t}-\delta f(u)_{x}=0
$$

the linear fourth order equation

$$
u_{t}+\gamma^{2} u_{x x x x}+u_{x x}=0,
$$

and the nonlinear diffusion equation

$$
u_{t}-\alpha\left(u^{3}\right)_{x x}=0,
$$

where $f(u)=u^{2} / 2$. We believe that this is the first time the equation is handled numerically using a fractional step method of this kind. The hyperbolic subproblem (1.6), and the parabolic subproblems (1.7) and (1.8), being of different natures, can be handled using different efficient numerical schemes. The convective equation (1.6) will be solved by some of the finite volume schemes designed for hyperbolic conservation laws (LeVeque, 2004) while the linear equation (1.7) will be handled by the Crank-Nicolson (CN) scheme and the second order Diagonally Implicit Runge-Kutta (DIRK) scheme. Equation (1.8) will be solved by an implicit/explicit conservative/non-conservative scheme. We highlight here that the split into (1.7) and (1.8) is motivated by the need to treat linear and nonlinear terms separately. This fractional step scheme converges to the solution of equation (1.1) and this can be shown following a similar analysis as given by Holden et al. (1999). For the specific case when $\delta=0$, we will combine only equations (1.7) and (1.8) to obtain the solution of the $\mathrm{CH}$ equation in (1.2). We highlight that extension to higher dimensions is feasible (Zachary et al., 1994), and will be addressed in our subsequent report.

The remaining part of this article is structured as follows: In $\S 2$ we give the description of the numerical schemes employed for each subproblem; the solution of equation (1.1) by the fractional step method is presented in $\S 3$; the solution properties are verified in $\S 4$ and we conclude in $\S 5$.

\section{Numerical Schemes}

We consider finite volume schemes to solve the $\mathrm{cCH}$ equation in (1.1). The notation adopted here is consistent with those known in the finite volume discretizations (see LeVeque (2004)). A uniform mesh, $x_{j+1 / 2}$ with fixed width $h$, where $x_{j}=j h, j \in \mathbb{Z}$ is considered. The standard time mesh is also employed, i.e., $t^{n}=n \Delta t$ with fixed time step size $\Delta t>0$. To take into account the discontinuity which may arise due to the hyperbolic equation in finite time, $v_{j}^{n}$ is considered as the approximation to the cell average of the true solution, thus,

$$
v_{j}^{n}=\frac{1}{h} \int_{x_{j-1 / 2}}^{x_{j+1 / 2}} u\left(x, t_{n}\right) d x .
$$

Given that $\mathcal{A}_{t}, \mathcal{L}_{t}$ and $\mathcal{N}_{t}$ are the exact solution operators of the convective, linear and the nonlinear equations (1.6), (1.7) and (1.8) respectively, and suppose the initial data is $v^{0}$. We introduce an auxiliary function $u_{\Delta t}$ so that the general fractional step approximate solution of the $\mathrm{cCH}$ equation follows the algorithm: 


\section{Algorithm 1}

$$
u_{\Delta t}(x, n \Delta t)=\left[\mathcal{L}_{\Delta t} \circ \mathcal{N}_{\Delta t} \circ \mathcal{A}_{\Delta t}\right]^{n} v^{0}(x),
$$

while a fractional step-splitting approximate solution of the $\mathrm{CH}$ equation (1.2) follows from the modification of the Algorithm 1 to give:

\section{Algorithm 2}

$$
u_{\Delta t}(x, n \Delta t)=\left[\mathcal{L}_{\Delta t} \circ \mathcal{N}_{\Delta t}\right]^{n} v^{0}(x)
$$

\subsection{Numerical Schemes for the Convection Equation}

The literature is rich with numerical schemes for equation (1.6). In most cases, the schemes are designed in such a way as to preserve the properties of the equation at the onset of discontinuity. For a comprehensive study on the properties of the equation and the schemes the reader is referred to, for example (Alexander and Tadmor, 2000; LeVeque, 2004), and the literatures therein. In this work we are interested in comparing the efficiency of three second order conservative explicit schemes namely: Non Staggered Central Difference (NSTG), Fully Discrete (FD) and the Weighted Essentially Non Oscillatory (WENO) schemes. A brief discussion on these schemes is given in the following subsections.

\subsubsection{Weighted Essentially Non-Oscillatory Scheme}

The scheme presented here follows the work of Shu (1997). The hyperbolic conservation law (1.6) can be cast into the semi-discrete form as

$$
\frac{d v_{j}}{d t}=A\left(v_{j}\right)
$$

where

$$
A\left(v_{j}\right)=-\frac{f_{j+1 / 2}-f_{j-1 / 2}}{h}
$$

The left hand side of the equation (2.4) will be handled by the third order Total Variation Diminishing Runge-Kutta scheme as developed in Shu and Osher (1988):

$$
\begin{gathered}
u^{1}=v^{n}+\Delta t A\left(v^{n}\right), \\
u^{2}=\frac{3}{4} v^{n}+\frac{1}{4} u^{1}+\frac{1}{4} \Delta t A\left(u^{1}\right), \\
v^{n+1}=\frac{1}{3} v^{n}+\frac{2}{3} u^{2}+\frac{2}{3} \Delta t A\left(u^{2}\right) .
\end{gathered}
$$

Remark 2.1 A Total Variation Diminishing scheme is one in which $T V\left(v^{n+1}\right) \leq T V\left(v^{n}\right)$ where $T V(u)$ is the total variation of the function, $u$. This type of scheme preserves monotonicity. 
Table 1: The values of $c_{r i}$

\begin{tabular}{|c|c|c|}
\hline $\mathrm{r}$ & $i=0$ & $i=1$ \\
\hline-1 & 1.5 & -0.5 \\
\hline 0 & 0.5 & 0.5 \\
\hline 1 & -0.5 & 1.5 \\
\hline
\end{tabular}

The discretized flux terms $f_{j \pm 1 / 2}$ are given as

$$
f_{j \pm 1 / 2}=g\left(v_{j \pm 1 / 2}^{-}, v_{j \pm 1 / 2}^{+}\right)
$$

where

$$
g(a, b)=\frac{1}{2}(f(a)+f(b)-\alpha(b-a)),
$$

is the Lax-Friedrich monotone flux and $\alpha=\max _{u}\left|f^{\prime}(u)\right|$ is a constant. The maximum is taken over the relevant range of $u$. The values of $v_{j+1 / 2}^{-}$and $v_{j-1 / 2}^{+}$are computed through the intercell average of the conserved variable $u$ as given in equation (2.1), i.e.,

$$
v_{j+1 / 2}^{-}=\sum_{r=0}^{k-1} \omega_{r} v_{j+1 / 2}^{r}, \quad v_{j+1 / 2}^{r}=\sum_{i=0}^{k-1} c_{r i} \bar{v}_{j-r+i}
$$

and

$$
v_{j-1 / 2}^{+}=\sum_{r=0}^{k-1} \tilde{\omega}_{r} v_{j-1 / 2}^{r}, v_{j-1 / 2}^{r}=\sum_{i=0}^{k-1} \tilde{c}_{r i} \bar{v}_{j-r+i} .
$$

where $r=0, \ldots, k-1$. The linear weights are

$$
\omega_{r}=\frac{\alpha_{r}}{\sum_{s=0}^{k-1} \alpha_{s}}, \alpha_{r}=\frac{d_{r}}{\left(\epsilon+\beta_{r}\right)^{2}},
$$

and

$$
\tilde{\omega}_{r}=\frac{\tilde{\alpha}_{r}}{\sum_{s=0}^{k-1} \tilde{\alpha}_{s}}, \quad \tilde{\alpha}_{r}=\frac{\tilde{d}_{r}}{\left(\epsilon+\beta_{r}\right)^{2}},
$$

where $\epsilon=10^{-6}, \beta_{r}$ are smooth indicators and $\tilde{d}_{r}=d_{k-1-r}$ are constants. The order of accuracy is $2 k-1$. We use $k=2$ in our computations, consequently, we only need $d_{0}=2 / 3$ and $d_{1}=1 / 3$. The values of constants $\tilde{c}_{r i}=c_{r-1, i}$ are given in Table 1 as extracted from Shu (1997). Also, the smooth indicators are given as

$$
\beta_{0}=\left(\bar{v}_{j+1}-\bar{v}_{j}\right)^{2}, \quad \beta_{1}=\left(\bar{v}_{j}-\bar{v}_{j-1}\right)^{2} .
$$

\subsubsection{Fully Discrete Scheme}

The fully discrete scheme for the convective equation (1.6) is given by (Alexander and Tadmor, 2000) as

$$
(u)_{j}^{n+1}=\frac{1}{h} \int_{x_{j-1 / 2}}^{x_{j+1 / 2}} \tilde{w}\left(\xi, t^{n+1}\right) d \xi=\lambda a_{j-1 / 2}^{n} w_{j-1 / 2}^{n+1}+\left(1-\lambda\left(a_{j-1 / 2}^{n}+a_{j+1 / 2}^{n}\right)\right) w_{j}^{n+1}
$$




$$
+\lambda a_{j+1 / 2}^{n} w_{j+1 / 2}^{n+1}+\frac{h}{2}\left(\left(\lambda a_{j-1 / 2}^{n}\right)^{2}\left(u_{x}\right)_{j-1 / 2}^{n+1}-\left(\lambda a_{j+1 / 2}^{n}\right)^{2}\left(u_{x}\right)_{j+1 / 2}^{n+1}\right),
$$

where $\lambda=\Delta t / h$ is the Courant-Friedrichs-Lewis (CFL) number, and

$$
\begin{gathered}
\left(u_{x}\right)_{j+1 / 2}^{n+1}=\frac{2}{h} \operatorname{minmod}\left(\frac{w_{j+1}^{n+1}-w_{j+1 / 2}^{n+1}}{1+\lambda\left(a_{j+1 / 2}^{n}-a_{j+3 / 2}^{n}\right)}, \frac{w_{j+1 / 2}^{n+1}-w_{j}^{n+1}}{1+\lambda\left(a_{j+1 / 2}^{n}-a_{j-1 / 2}^{n}\right)}\right), \\
w_{j+1 / 2}^{n+1}=\frac{u_{j}^{n}+u_{j+1}^{n}}{2}+\frac{h-a_{j+1 / 2}^{n} \Delta t}{4}\left(\left(u_{x}\right)_{j}^{n}-\left(u_{x}\right)_{j+1}^{n}\right)-\frac{1}{2 a_{j+1 / 2}^{n}}\left(f\left(u_{j+1 / 2, r}^{n+1 / 2}\right)-f\left(u_{j+1 / 2, l}^{n+1 / 2}\right)\right), \\
w_{j}^{n+1}=u_{j}^{n}+\frac{\Delta t}{2}\left(a_{j-1 / 2}^{n}-a_{j+1 / 2}^{n}\right)\left(u_{x}\right)_{j}^{n}-\frac{\lambda}{1-\lambda\left(a_{j-1 / 2}^{n}+a_{j+1 / 2}^{n}\right)}\left(f\left(u_{j+1 / 2, l}^{n+1 / 2}\right)-f\left(u_{j-1 / 2, r}^{n+1 / 2}\right)\right),
\end{gathered}
$$

with

$$
\begin{gathered}
u_{j+1 / 2, l}^{n+1 / 2}=u_{j+1 / 2, l}^{n}-\frac{\Delta t}{2} f\left(u_{j+1 / 2, l}^{n}\right)_{x}, \quad u_{j+1 / 2, l}^{n}=u_{j}^{n}+h\left(u_{x}\right)_{j}^{n}\left(\frac{1}{2}-\lambda a_{j+1 / 2}^{n}\right), \\
u_{j+1 / 2, r}^{n+1 / 2}=u_{j+1 / 2, r}^{n}-\frac{\Delta t}{2} f\left(u_{j+1 / 2, r}^{n}\right)_{x}, \quad u_{j+1 / 2, r}^{n}=u_{j+1}^{n}-h\left(u_{x}\right)_{j+1}^{n}\left(\frac{1}{2}-\lambda a_{j+1 / 2}^{n}\right) .
\end{gathered}
$$

The local speed of propagation is

$$
a_{j+1 / 2}^{n}=\max \left[\rho\left(\frac{\partial f}{\partial u}\left(u_{j+1 / 2}^{-}\right)\right), \rho\left(\frac{\partial f}{\partial u}\left(u_{j+1 / 2}^{+}\right)\right)\right],
$$

where $\rho$ is the spectral radius,

$$
u_{j+1 / 2}^{+}=u_{j+1}^{n}-\frac{h}{2}\left(u_{x}\right)_{j+1}^{n}, u_{j+1 / 2}^{-}=u_{j}^{n}+\frac{h}{2}\left(u_{x}\right)_{j}^{n}
$$

and

with

$$
\left(u_{x}\right)_{j}^{n}=\operatorname{minmod}\left(\frac{u_{j}^{n}-u_{j-1}^{n}}{h}, \frac{u_{j+1}^{n}-u_{j}^{n}}{h}\right) .
$$

$$
\operatorname{minmod}(a, b)=\frac{\operatorname{sgn}(a)+\operatorname{sgn}(b)}{2} \min (|a|,|b|) .
$$

\subsubsection{Non Staggered Central Difference Scheme}

This scheme as given in (Tadmor and Nessyahu, 1990) solves equation (1.6) via a predictorcorrector formulation,

$$
\begin{gathered}
v_{j}^{n+1 / 2}=v_{j}^{n}-\frac{\lambda f_{j}^{\prime}}{2} \\
v_{j}^{n+1}=\frac{1}{2}\left(v_{j+1}^{n}+v_{j-1}^{n}\right)+\frac{1}{4}\left(v_{j-1}^{\prime n}-v_{j+1}^{\prime n}\right)-\frac{\lambda}{2}\left(f\left(v_{j+1}^{n+1 / 2}\right)-f\left(v_{j-1}^{n+1 / 2}\right)\right) .
\end{gathered}
$$

The non oscillatory behavior of this scheme is guaranteed by the choice

$$
v_{j}^{\prime}=\operatorname{minmod}\left(\Delta v_{j+1 / 2}, \Delta v_{j-1 / 2}\right) \text {, }
$$

and

$$
f_{j}^{\prime}=a\left(v_{j}\right) v_{j}^{\prime}
$$

where $a\left(v_{j}\right)$ is the derivative of the flux function with respect to the argument $v_{j}$. 


\subsection{Numerical Schemes for the nonlinear equation}

We solve the nonlinear diffusion equation (1.8) explicitly and implicitly by considering the scheme as second order conservative in which case the equation is written as

$$
u_{t}=\alpha\left(u^{3}\right)_{x x}
$$

and discretized using the $\theta$ method to give

$$
v_{j}^{n+1}=v_{j}^{n}+r\left(\theta\left[\phi_{j+1}^{n+1}-2 \phi_{j}^{n+1}+\phi_{j-1}^{n+1}\right]+(1-\theta)\left[\phi_{j+1}^{n}-2 \phi_{j}^{n}+\phi_{j-1}^{n}\right]\right),
$$

where $\phi_{j}^{n}=\left(v_{j}^{n}\right)^{3}, r=\frac{\Delta t}{h^{2}}$ and $v_{j}^{n}$ is the intercell averages as given in equation (2.1). For the purpose of comparison, we also solve (1.8) with second order non conservative schemes, in which case the equation is re-written as

$$
u_{t}=\alpha\left(\varphi u_{x}\right)_{x}
$$

where $\varphi=3 u^{2}$. Similarly, this is discretized using the $\theta$ method to give

$$
\begin{array}{r}
v_{j}^{n+1}=v_{j}^{n}+r \theta\left(\varphi_{j+1 / 2}^{n+1}\left[v_{j+1}^{n+1}-v_{j}^{n+1}\right]-\varphi_{j-1 / 2}^{n+1}\left[v_{j}^{n+1}-v_{j-1}^{n+1}\right]\right)+ \\
r(1-\theta)\left(\varphi_{j+1 / 2}^{n}\left[v_{j+1}^{n}-v_{j}^{n}\right]-\varphi_{j-1 / 2}^{n}\left[v_{j}^{n}-v_{j-1}^{n}\right]\right),
\end{array}
$$

where

$$
\varphi_{j \pm 1 / 2}=\frac{\varphi_{j \pm 1}+\varphi_{j}}{2}
$$

and $\theta$ is a weighting factor. The schemes (2.12) and (2.14) will be fully implicit if $\theta=1$, Crank-Nicolson if $\theta=0.5$ and fully explicit when $\theta=0$. The explicit schemes result in a system of linear equations while the implicit schemes give a system of nonlinear equations. While the linear equations can be solved directly, the nonlinear equations are solved by the iterative Newton-Raphson method. Coupled with the corresponding second order boundary conditions, the expected rate of convergence is 2 for all these schemes.

\subsubsection{Convergence of the nonlinear term}

Here we consider the following example

\section{Problem 1}

$$
\left\{\begin{array}{l}
u_{t}-\left(u^{3}\right)_{x x}=f(x), x \in[0, \pi] \\
u(0, t)=u(\pi, t)=0 \\
u(x, 0)=\sin (x)
\end{array}\right.
$$

where $f(x)=-\frac{3}{4}[3 \sin 3 x-\sin x]$, to test the effectiveness of schemes (2.12) and (2.14). We compare our results with the exact steady state solution $u(x, t)=\sin (x)$ at time $T=0.5$. The $\ell^{\infty}$ error, $e\left(m_{i}\right)$, for different number of grid points, $m_{i}$, were computed and the convergent rates, $p$, were calculated based on the relation

$$
p=\frac{\log \left(e\left(m_{2}\right) / e\left(m_{1}\right)\right)}{\log \left(m_{2} / m_{1}\right)} .
$$

The errors and the convergent rates due to each of the scheme are given in Table 2 for different grid points. It is obvious that all the schemes are equally good. 
Table 2: Convergence rate of the schemes for the nonlinear equation (1.8)

\begin{tabular}{|c|c|c|c|c|}
\hline Grid points & \multicolumn{4}{|c|}{ maximum absolute error $\times 10^{-3}(\mathrm{p})$ at $\mathrm{T}=0.5$} \\
\cline { 2 - 5 } & \multicolumn{2}{|c|}{ Conservative } & Non Conservative Schemes \\
\cline { 2 - 5 } & CN & Explicit & CN & Explicit \\
\hline 20 & 18.1 & 18.2 & 23.0 & 20.0 \\
\hline 40 & $6.34(1.46)$ & $6.39(1.46)$ & $7.86(1.49)$ & $7.30(1.50)$ \\
\hline 80 & $2.03(1.62)$ & $2.02(1.63)$ & $2.50(1.63)$ & $2.39(1.64)$ \\
\hline 160 & $0.58(1.78)$ & $0.58(1.78)$ & $0.72(1.78)$ & $0.70(1.78)$ \\
\hline
\end{tabular}

\subsection{Numerical Scheme for the linear equation}

We employ the second order Crank-Nicolson and the DIRK scheme to solve equation (1.7). In both cases, the corresponding discrete problem is a system of linear algebraic equations which can be solved by direct methods. We note that the presence of the parameter $\gamma$ in (1.7) makes the problem stiff as $\gamma \rightarrow 0$. The CN scheme, through the $\theta$ method, can be expressed as

$$
v_{i}^{n+1}-\theta f\left(v_{i}^{n+1}\right)=v_{i}^{n}+(1-\theta) f\left(v_{i}^{n}\right),
$$

while the DIRK scheme is

$$
\begin{aligned}
v_{i}^{*} & =v_{i}^{n-1}+\frac{1}{2} f\left(v_{i}^{*}\right), \\
v_{i}^{n} & =v_{i}^{n-1}+f\left(v_{i}^{*}\right),
\end{aligned}
$$

where

$$
f\left(v_{i}^{n}\right)=-r\left(v_{i-1}^{n}-2 v_{i}^{n}+v_{i+1}^{n}\right)-\gamma^{2} \mu\left(v_{i-2}^{n}-4 v_{i-1}^{n}+6 v_{i}^{n}-4 v_{i+1}^{n}+v_{i+2}^{n}\right)
$$

and $\mu=\frac{\Delta t}{h^{4}}$. A similar analysis as in Ternat et al. (2011) is done for both the DIRK and the CN schemes. For brevity we skip the details of this analysis and only compare their performance via an example. We solve equation (1.7) as an initial boundary value problem as follows

\section{Problem 2}

$$
\left\{\begin{array}{l}
u_{t}+\left(u+\gamma^{2} u_{x x}\right)_{x x}=0, \quad x \in(0,2 \pi) \\
u(x, 0)=\cos (x / \gamma), \\
u(0, t)=1, \quad u_{x}(0, t)=0 \\
u(2 \pi, t)=\cos (2 \pi / \gamma), \quad u_{x}(2 \pi, t)=-\sin (2 \pi / \gamma) / \gamma
\end{array}\right.
$$

The numerical solutions are compared with the exact solution $u(x, t)=\cos (x / \gamma)$. The Table 3 below shows the comparison of performance of the two methods for different values of $\gamma$.

It is worth highlighting here that for $\gamma^{2}=0.1$, it took the DIRK scheme less than 0.0025 seconds and the CN scheme less than 0.0027 seconds of computing time to produce each of the results in Table 3. Both schemes were even faster and clocked the same computer time when $\gamma^{2}$ was increased to $\gamma^{2}=1$. Results in Table 3 also confirms that the schemes are 
Table 3: Convergence rate of the linear equation

\begin{tabular}{|c|c|c|c|c|}
\hline \multirow{2}{*}{ Grid points } & \multicolumn{3}{|c|}{ maximum absolute error $\times 10^{-4}$ (convergence rate) at $\mathrm{T}=0.5$} \\
\cline { 2 - 5 } & \multicolumn{2}{|c}{$\gamma^{2}=1$} & \multicolumn{2}{c|}{$\gamma^{2}=.1$} \\
\cline { 2 - 5 } & $\mathrm{CN}$ & $\mathrm{DIRK}$ & $\mathrm{CN}$ & $\mathrm{DIRK}$ \\
\hline 20 & 147 & 163 & 4632 & 5506 \\
\hline 40 & $37.6(1.90)$ & $38.9(1.99)$ & $1092(2.01)$ & $1155(2.17)$ \\
\hline 80 & $9.61(1.93)$ & $9.77(1.96)$ & $299(1.83)$ & $306(1.88)$ \\
\hline 160 & $2.36(2.01)$ & $2.38(2.02)$ & $69.8(2.08)$ & $70.7(2.10)$ \\
\hline
\end{tabular}

comparable in terms of their performance and convergence. But because of the observed time step restrictions of the CN scheme for such stiff problems, DIRK scheme will be employed throughout this work for the solution of the linear equation (1.7).

Remark 2.2 From here forthwith, we will refer to the schemes employed to solve the cCH equation by the name of the scheme used to solve the nonlinear hyperbolic part of the full equation.

\section{Solution of the full equation}

In this section we discuss the solution of equation (1.1) for the two main cases: when $\delta=0$ and when $\delta \neq 0$, for different choices of $\alpha$. All the simulations were run on a Windows XP, Intel Core 2 Duo, 2GB RAM desktop and all the solutions were achieved within 10 seconds of computing time.

\subsection{The Cahn-Hilliard equation}

In this section we are interested in providing a numerical solution to the traditional $\mathrm{CH}$ equation by the fractional splitting method. For the analysis we consider

\section{Problem 3}

$$
\left\{\begin{array}{l}
u_{t}+\left(\alpha u^{3}+u+\gamma^{2} u_{x x}\right)_{x x}=0, \quad x \in(0,6) \\
u(x, 0)=\cos (\pi x / 6),
\end{array}\right.
$$

and boundary conditions (1.5).

It is obvious that the solution operator of this equation can be split into the solution operator of equation (1.8) and the solution operator of equation (1.7), and Algorithm 2 can be used. We determine the spatial rate of convergence of this scheme for the Cahn-Hilliard equation (3.1) for $\gamma=\alpha=1$ using the refined grid as the exact solution. The rate of convergence was computed by employing the equation (2.16) and the results are presented in Table 4 .

In addition, we note that the evolution of equation (3.1), for $\alpha=1 / 3$ and $\gamma^{2}=0.02$, shown in Figure 1 compares well with the work of Elliott and French (1987). 
Table 4: Convergence rate of the fractional step for the Cahn-Hilliard Equation

\begin{tabular}{|c|c|c|c|c|}
\hline Grid points & \multicolumn{4}{|c|}{ maximum absolute error $\times 10^{-3}(\mathrm{p})$ at $\mathrm{T}=1$} \\
\cline { 2 - 5 } & \multicolumn{2}{|c|}{ Conservative } & \multicolumn{1}{c|}{ Non Conservative Schemes } \\
\cline { 2 - 5 } & CN & Explicit & CN & Explicit \\
\hline 20 & 98.8 & 102 & 102 & 101 \\
\hline 40 & $45.9(1.1)$ & $47.5(1.1)$ & $47.5(1.1)$ & $47.2(1.1)$ \\
\hline 80 & $19.6(1.21)$ & $20.3(1.2)$ & $20.4(1.2)$ & $20.2(1.2)$ \\
\hline 160 & $6.52(1.6)$ & $6.74(1.6)$ & $6.78(1.6)$ & $6.73(1.6)$ \\
\hline
\end{tabular}

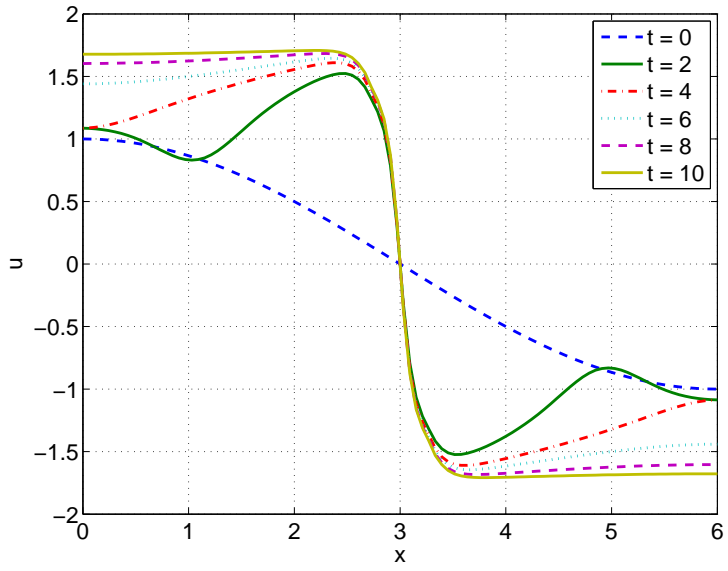

(a) Evolution in time

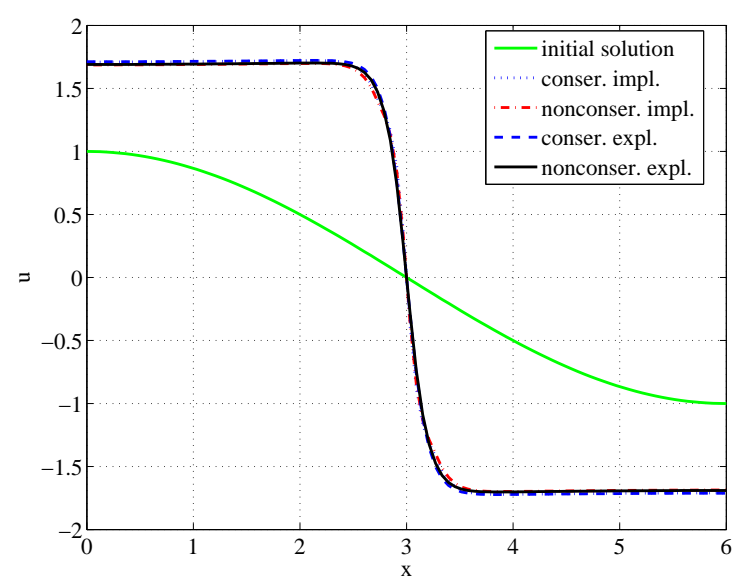

(b) Schemes comparison

Figure 1: Solution of the Cahn-Hilliard (3.1) at time $T=10$. Profiles in (a) are given at equally spaced time intervals.

\subsection{The Convective Cahn-Hilliard equation}

In this section we give the solution of the $\mathrm{cCH}$ equation (1.1) using the splitting Algorithm 1. In particular, we consider

\section{Problem 4}

$$
\left\{\begin{array}{l}
u_{t}-\delta u u_{x}+\left(u+\alpha u^{3}+\gamma^{2} u_{x x}\right)_{x x}=0 \\
u(x, 0)=-\sin (x / 6)
\end{array}\right.
$$

and boundary conditions (1.5).

For the purpose of comparison with the literature, we choose $\gamma=\alpha=1$ and $\delta=0.1-$ see for example (Emmott and Bray, 1996; Watson, 2003). Following the same refined-grid method as discussed for the Cahn-Hilliard equation, the convergence rate was computed and the results are shown in Tables 5 to 7 . As expected, all schemes predict a second order convergence in space.

The profiles of the solution as compared with the exact steady state solution are also given in Figures 2 and 3. For all the schemes, the CFL number is taken between 0.1 and 0.5 which also gives a time step within the stability restriction of the explicit schemes for the 
Table 5: Convergence rate of the fractional step for the cCH equation with the NSTG scheme.

\begin{tabular}{|c|c|c|c|c|}
\hline Grid points & \multicolumn{4}{|c|}{ maximum absolute error $\times 10^{-3}$ (convergence rate) at $\mathrm{T}=1$} \\
\cline { 2 - 5 } & \multicolumn{2}{|c|}{ Conservative } & \multicolumn{2}{c|}{ Non Conservative Schemes } \\
\cline { 2 - 5 } & CN & Explicit & CN & Explicit \\
\hline 20 & 116 & 116 & 116 & 116 \\
\hline 40 & $53.6(1.08)$ & $53.5(1.08)$ & $53.6(1.07)$ & $53.4(1.08)$ \\
\hline 80 & $22.9(1.21)$ & $22.8(1.21)$ & $22.8(1.21)$ & $22.8(1.20)$ \\
\hline 160 & $7.72(1.55)$ & $7.70(1.55)$ & $7.72(1.55)$ & $7.70(1.55)$ \\
\hline
\end{tabular}

Table 6: Convergence rate of the fractional step for the cCH equation with the WENO scheme.

\begin{tabular}{|c|c|c|c|c|}
\hline Grid points & \multicolumn{4}{|c|}{ maximum absolute error $\times 10^{-3}$ (convergence rate) at T = 1} \\
\cline { 2 - 5 } & \multicolumn{2}{|c|}{ Conservative } & \multicolumn{2}{c|}{ Non Conservative Schemes } \\
\cline { 2 - 5 } & CN & Explicit & CN & Explicit \\
\hline 20 & 110 & 110 & 110 & 110 \\
\hline 40 & $51.5(1.05)$ & $51.4(1.06)$ & $51.5(1.05)$ & $51.3(1.06)$ \\
\hline 80 & $22.2(1.20)$ & $22.1(1.20)$ & $22.2(1.20)$ & $22.1(1.20)$ \\
\hline 160 & $7.58(1.54)$ & $7.55(1.54)$ & $7.57(1.54)$ & $7.55(1.54)$ \\
\hline
\end{tabular}

Table 7: Convergence rate of the fractional step for the $\mathrm{cCH}$ equation with the FD scheme.

\begin{tabular}{|c|c|c|c|c|}
\hline Grid points & \multicolumn{4}{|c|}{ maximum absolute error $\times 10^{-3}$ (convergence rate) at $\mathrm{T}=1$} \\
\cline { 2 - 5 } & \multicolumn{2}{|c|}{ Conservative } & \multicolumn{2}{c|}{ Non Conservative Schemes } \\
\cline { 2 - 5 } & CN & Explicit & CN & Explicit \\
\hline 20 & 110 & 110 & 110 & 110 \\
\hline 40 & $51.6(1.06)$ & $51.5(1.06)$ & $51.6(1.05)$ & $51.4(1.06)$ \\
\hline 80 & $22.2(1.20)$ & $22.1(1.20)$ & $22.2(1.19)$ & $22.1(1.20)$ \\
\hline 160 & $7.58(1.54)$ & $7.56(1.54)$ & $7.57(1.54)$ & $7.56(1.54)$ \\
\hline
\end{tabular}

nonlinear equation. Figures 3 highlights the poor performance of explicit schemes (for the nonlinear equation) when coupled by the NSTG scheme (for the advection equation).

\section{Solution properties}

Here we will first validate some of the known properties of the Cahn-Hilliard equation and end this section by validating the properties of the convective Cahn-Hilliard equation as documented in the literature. 


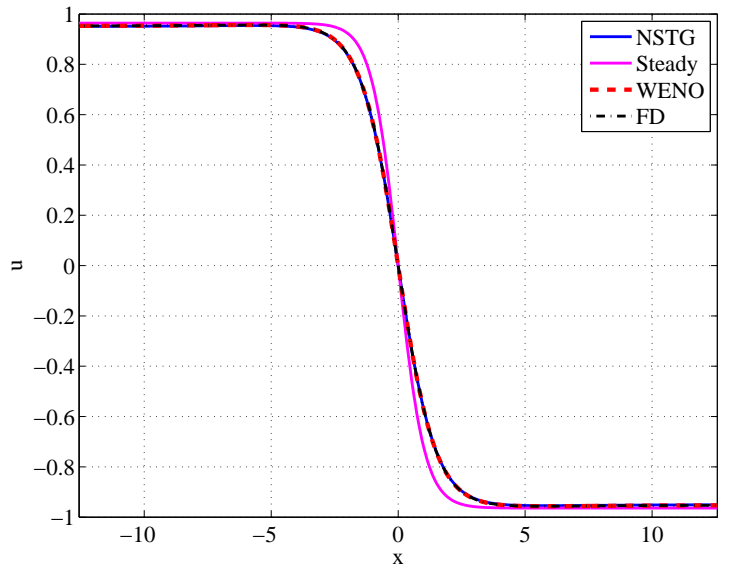

(a) Conservative implicit schemes

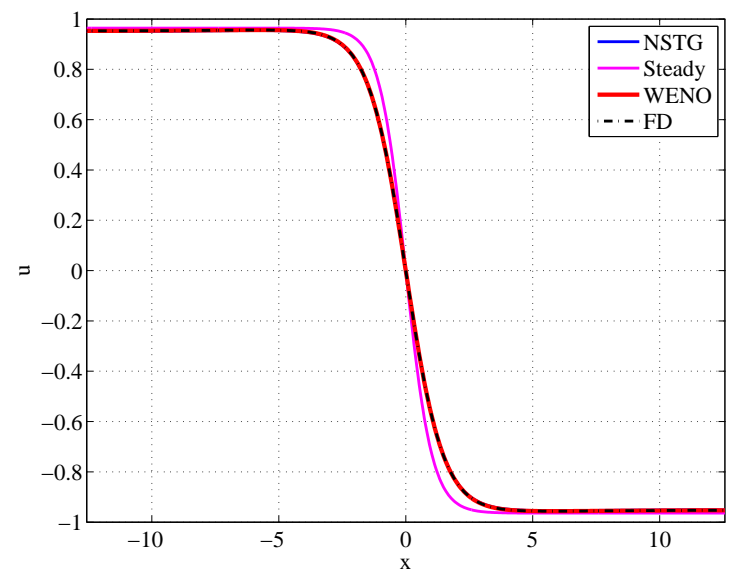

(b) Non-Conservative implicit schemes

Figure 2: Numerical solution of the convective Cahn-Hilliard equation.

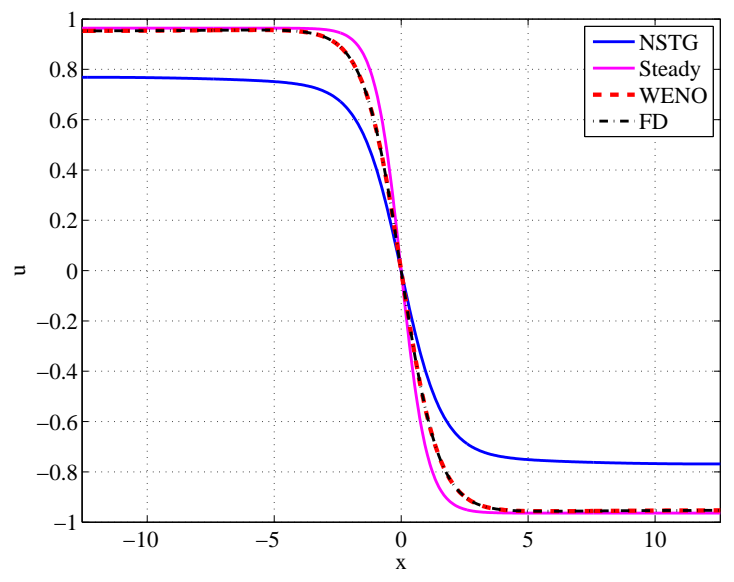

(a) Conservative explicit schemes

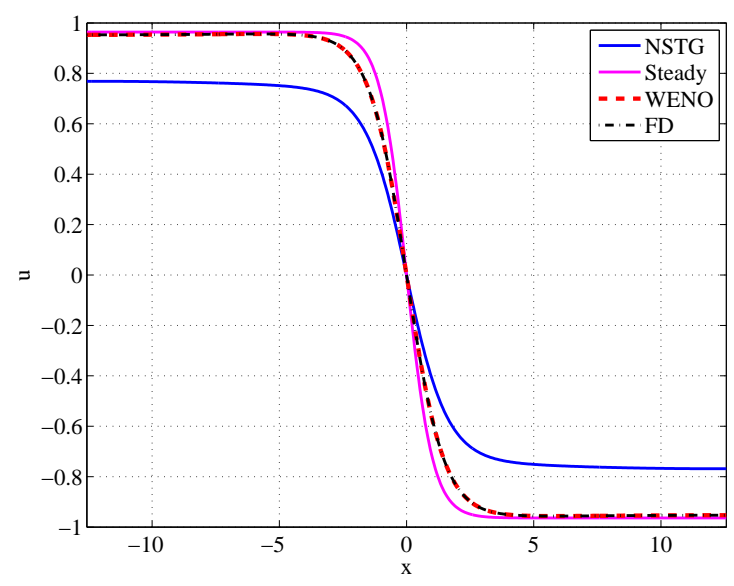

(b) Non-Conservative explicit schemes

Figure 3: Numerical solution of the convective Cahn-Hilliard equation.

\subsection{The Cahn-Hilliard equation}

It has been documented that the solution provided by any numerical scheme that solves the Cahn-Hilliard equation must satisfy two main properties (De Mello and Silveira Filho, 2005; Dehghan and Mirzaei, 2009; Elliott and French, 1987; Novick-Cohen and Segel, 1984):

1. Conservation of mass:

$$
\int_{\Omega} u_{0}(x) d x=\int_{\Omega} u d x=M L, t>0
$$

where $M$ is the total mass of the system and

2. Dissipation of the Ginzburg-Landau free energy functional (equation (1.4)) as time passes. 


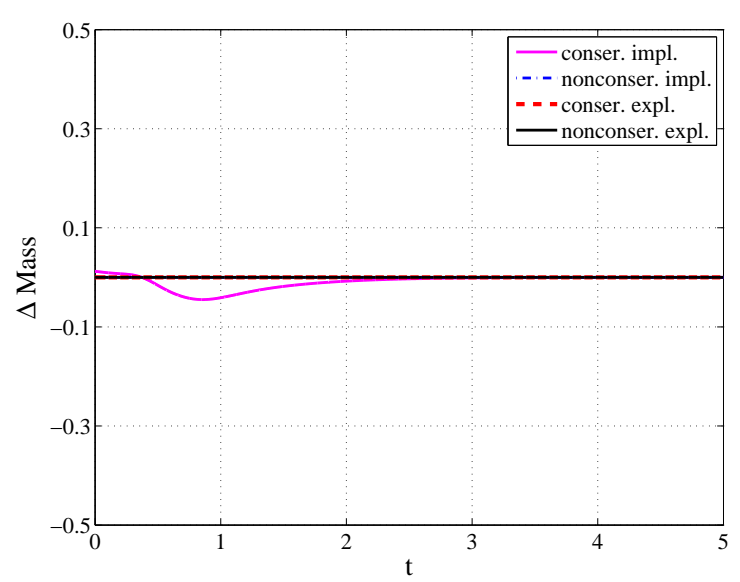

(a) Conservation of Mass $\times 10^{-4}$

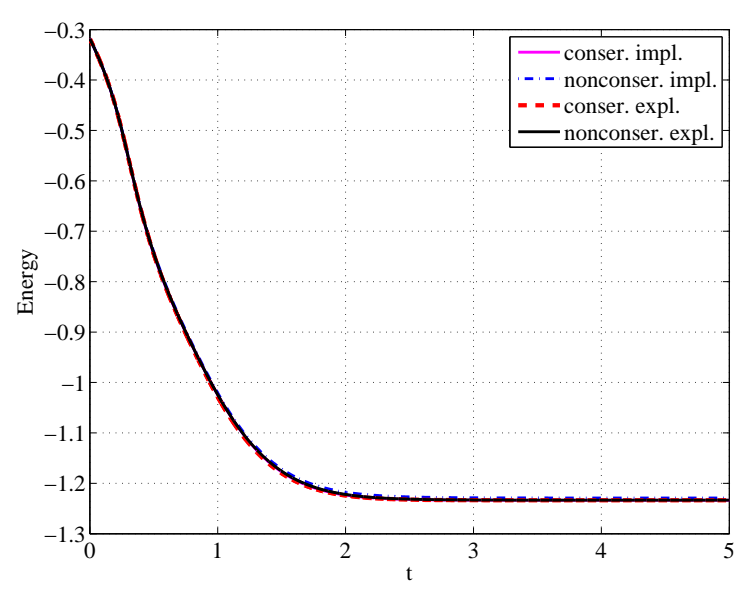

(b) Dissipation of Energy

Figure 4: Conservation of mass and dissipation of energy.

Hence, this section is dedicated to validate these two main properties using Algorithm 2 and Problem 3. The profiles in Figure 4(a) verifies the conservation of mass while Figure 4(b) validates the dissipation of energy as expected (see for example, Dehghan and Mirzaei (2009); Elliott and French (1987)). Note that most of the profiles for the different schemes overlap and hence cannot be distinguished from each other. The mass profile when the conservative implicit scheme is applied to the nonlinear subproblem shows some deviation from the other schemes.

\subsection{The Convective Cahn-Hilliard equation}

We will conclude this work by showing the conservation of mass and the coarsening property for the convective Cahn-Hilliard equation (1.1). Coarsening is a major property since it is the behavior of the physical process (spinodal decomposition) that the equation models (Golovin et al., 1998; Podolny et al., 2005; Watson, 2003) as time progresses. Here we solve (see for example Eden and Kalantarov (2007)),

\section{Problem 5}

$$
\left\{\begin{array}{l}
u_{t}-\delta u u_{x}+\left(u+\alpha u^{3}+\gamma^{2} u_{x x}\right)_{x x}=0, x \in[-4 \pi, 4 \pi] \\
u(x, 0)=0.5 \cos x \\
u(x+L, t)=u(x, t)
\end{array}\right.
$$

subject to periodic boundary conditions,

for $\delta=0.1$ using 160 grid points and Algorithm 1. The integration continues until the solution coarsens and below we present a sequence of figures (i.e., Figures 5 and 6) showing the behavior of the solution over time. 


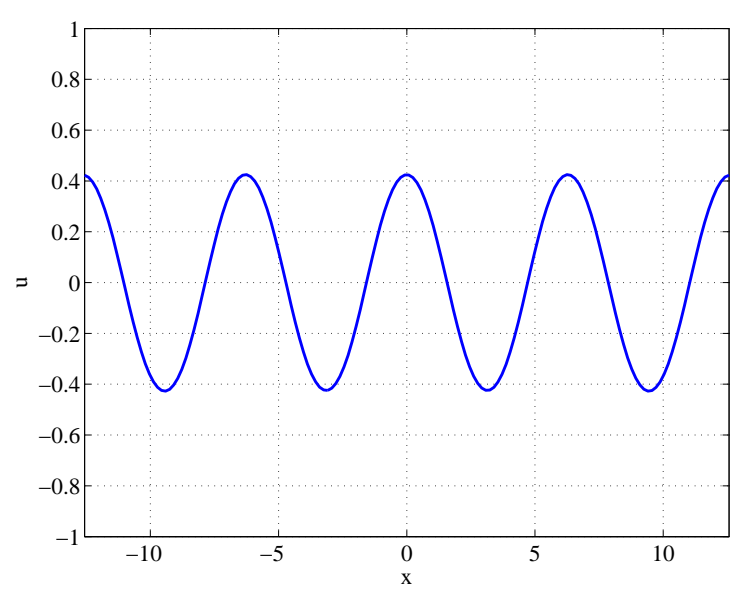

(a) $T=1$ (Formation of periodic structure)

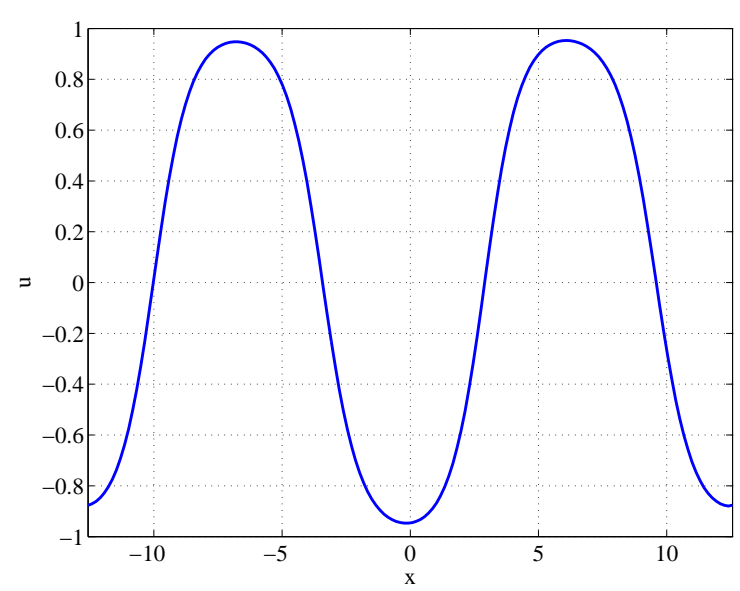

(b) $T=50$

Figure 5: Coarsening process.

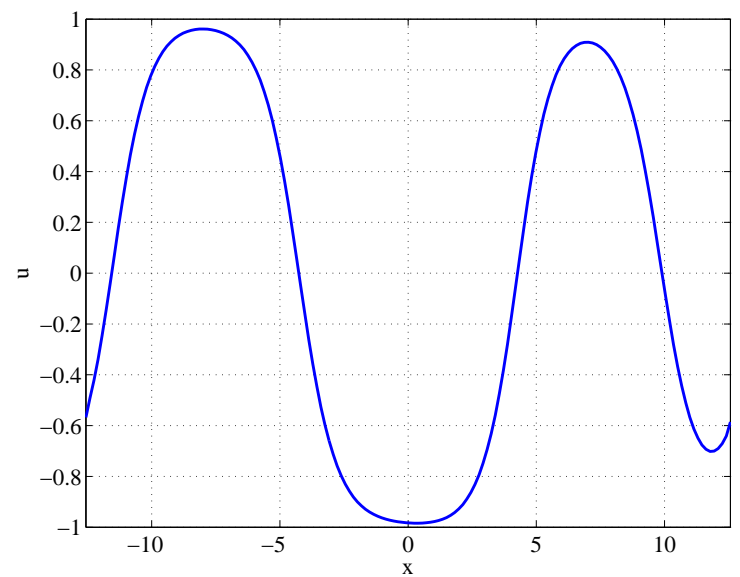

(a) $T=320$

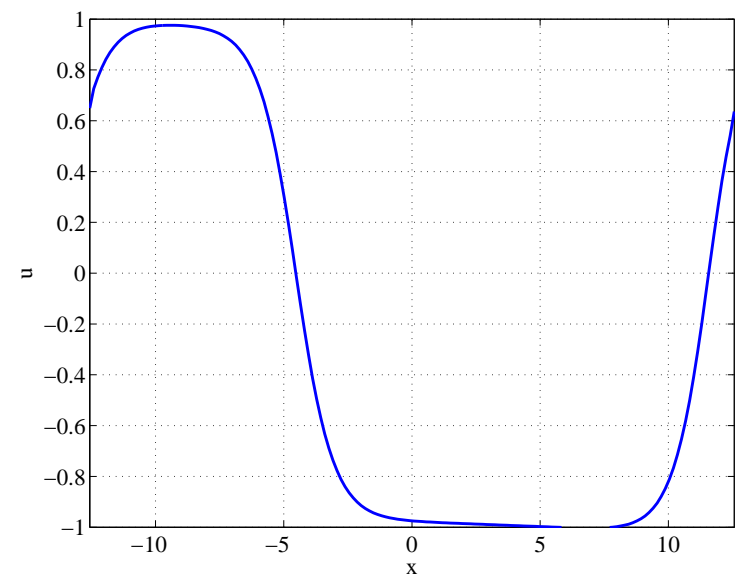

(b) $T=640$ (Kink-anti-kink pair)

Figure 6: Coarsening process.

For the conservation of mass, we have:

$$
\begin{aligned}
\frac{d}{d t} \int_{-4 \pi}^{4 \pi} u d x & =\int_{-4 \pi}^{4 \pi} u_{t} d x \\
& =\int_{-4 \pi}^{4 \pi}\left(\delta \frac{u^{2}}{2}+\left(-\gamma^{2} u_{x x}+\phi(u)\right)_{x}\right)_{x} d x \\
& =\left.\left(\delta \frac{u^{2}}{2}+\left(-\gamma^{2} u_{x x}+\phi(u)\right)_{x}\right)\right|_{-4 \pi} ^{4 \pi}=0
\end{aligned}
$$

The last equality is due to periodicity.

Figures 7 and 8 shows the conservation of the mass for all the schemes as expected. 


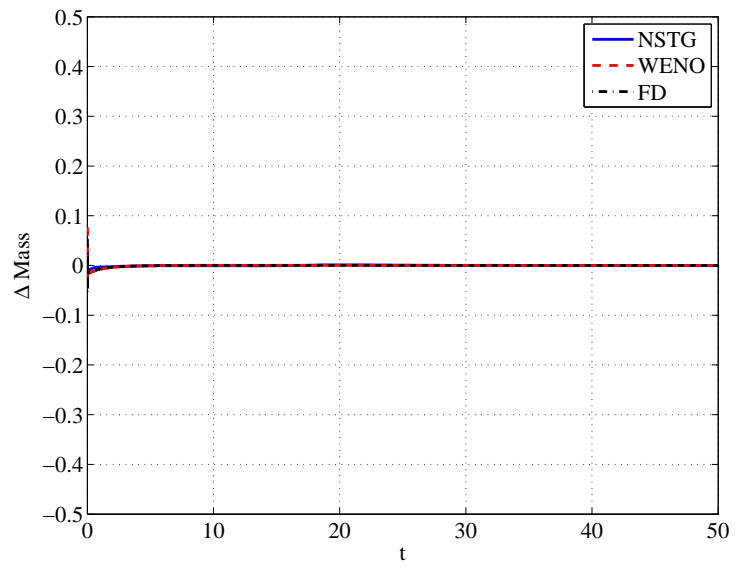

(a) Conservative Implicit Schemes

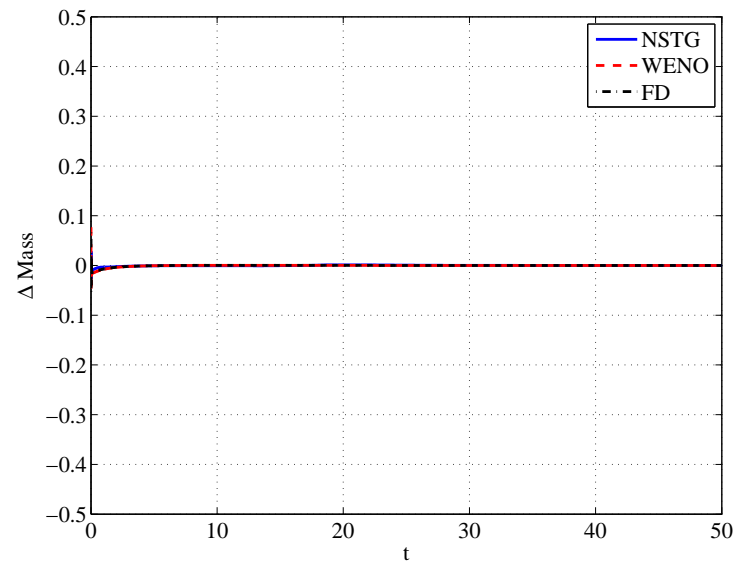

(b) Non-Conservative Implicit Schemes

Figure 7: Conservation of mass .

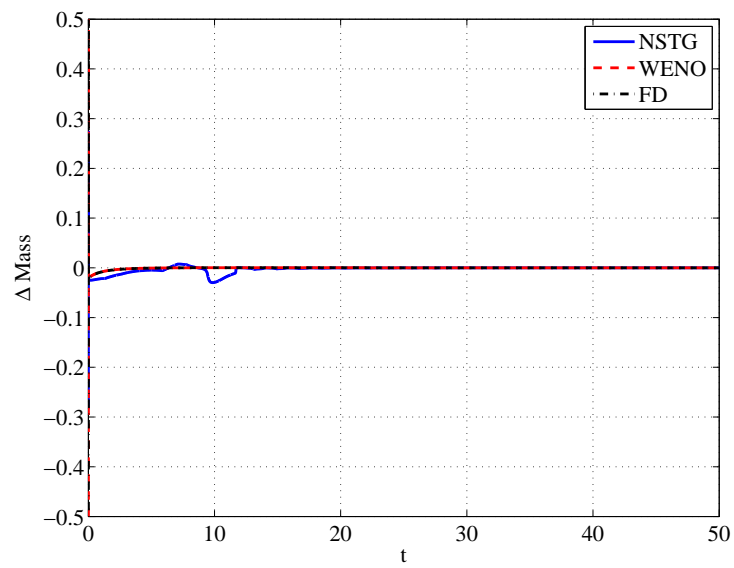

(a) Conservative Explicit Schemes

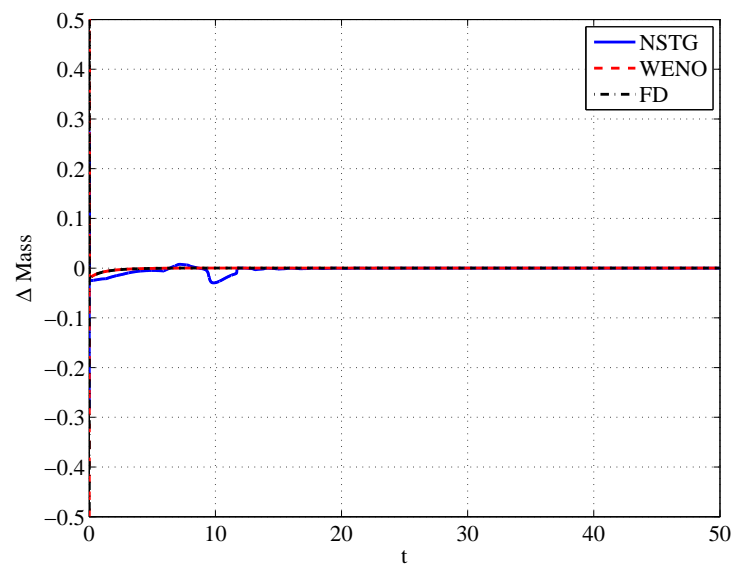

(b) Non-Conservative Explicit Schemes

Figure 8: Conservation of mass.

\subsubsection{From coarsening to roughening}

Another important property of the $\mathrm{cCH}$ equation (1.1) is transition from roughening to an orderless pattern. This is due to the fact that as the value of the driving force, $\delta$, increases, the convective term dominates the equation, consequently it behaves exactly like the famous Kuramoto-Sivashinsky equation. This can be verified by taking $u \rightarrow u / \delta$ in equation (1.1), then take $\delta \rightarrow \infty$. This was noted by Golovin et al. (Golovin et al., 2001) and Watson (Watson, 2003) among others. The former verified this property using the stationary and the traveling wave solution of equation (1.1) while the latter just remarked this behavior. Here, Figure (9), the solution profile of Problem (5) at final computation time $T=80$ with $\delta=5$, clearly shows this behavior. 


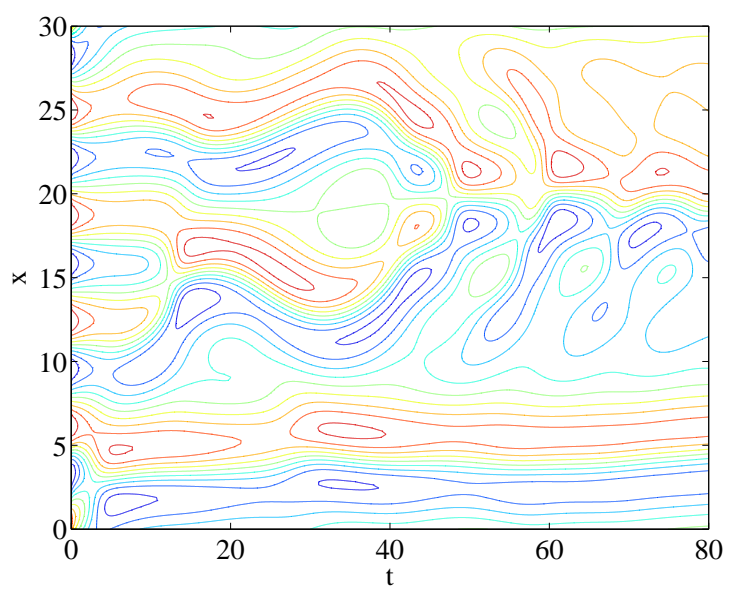

Figure 9: Rough behavior of the convective Cahn-Hilliard equation.

\section{Conclusion}

In this work we presented a finite difference approximation for the solution of the convective Cahn-Hilliard equation using a step splitting method. Several schemes (WENO, NSTG and the FD) were used to handle the convective hyperbolic term and the WENO and NSTG schemes perform better when combined with implicit schemes than with explicit schemes for the nonlinear term. The deviation of the solution from the steady state can clearly be seen when the NSTG scheme is used.

In addition, we tested the presented method by validating the dissipation of energy and the mass conservation properties of the solution. In all cases, a good agreement with the literature was observed. In particular, the FD scheme outperformed the other schemes.

\section{Acknowledgement}

We are sincerely grateful to the three anonymous reviewers whose extensive and thoughtful comments led to significant improvements in this paper.

\section{References}

Alexander, K. and Tadmor, E. (2000). New high-resolution central schemes for nonlinear conservation laws and convection-diffusion equations. Journal of Computational Physics, $160: 241-282$.

Christlieb, A., Jones, J., Promislow, K., Wetton, B., and Willoughby, M. (2012). High accuracy solutions to energy gradient flows from material science models. Submitted to Journal of Computaional Physics.

Cueto-Felgueroso, L. and Peraire, J. (2008). A time-adaptive finite volume method for the Cahn-hilliard and Kuramoto-Sivashinsky equations. Journal of Computational Physics, 227(24):9985-10017. 
De Mello, E. and Silveira Filho, O. (2005). Numerical study of the Cahn-Hilliard equation in one, two and three dimensions. Physica A: Statistical Mechanics and its Applications, 347:429-443.

Dehghan, M. and Mirzaei, D. (2009). A numerical method based on the boundary integral equation and dual reciprocity methods for one-dimensional Cahn-Hilliard equation. Engineering Analysis with Boundary Elements, 33(4):522-528.

Du, Q. and Nicolaides, R. (1991). Numerical analysis of a continuum model of phase transition. SIAM Journal on Numerical Analysis, pages 1310-1322.

Eden, A. and Kalantarov, V. (2007). The convective Cahn-Hilliard equation. Applied Mathematics Letters, 20(4):455-461.

Elliott, C. and French, D. (1987). Numerical studies of the Cahn-Hilliard equation for phase separation. IMA Journal of Applied Mathematics, 38:97-128.

Elliott, C. and French, D. (1989). A nonconforming finite-element method for the twodimensional Cahn-Hilliard equation. SIAM Journal on Numerical Analysis, 26(4):884903.

Elliott, C. and Songmu, Z. (1986). On the Cahn-Hilliard equation. Archive for Rational Mechanics and Analysis, 96(4):339-357.

Emmott, C. and Bray, A. (1996). Coarsening dynamics of a one dimensional driven CahnHilliard equation. Physical Review E, 54(5):4568-4575.

Eyre, D. (1998). Unconditionally gradient stable time marching the Cahn-Hilliard equation. In MRS Proceedings, volume 529. Cambridge Univ Press.

Golovin, A., Davis, S., and Nepomnyashchy, A. (1998). A convective Cahn-Hilliard model for the formation of facets and corners in crystal growth. Physica D: Nonlinear Phenomena, 122(1-4):202-230.

Golovin, A., Nepomnyashchy, A., Davis, S., and MA, Z. (2001). Convective Cahn-Hilliard models: From coarsening to roughening. Physical Review Letters, 86(8):1550-1553.

Heldge, H., Kenneth, H., Knut-Andreas, L., and Nils, H. (2010). Splitting methods for partial differential equations with rough solutions. European Mathematical Society series of lectures in Mathematics.

Holden, H., Karlsen, K., and Risebro, N. (1999). Operator splitting methods for generalized Korteweg-de Vries equations. Journal of Computational Physics, 153(1):203-222.

Lee, H., Choi, J., and Kim, J. (2011). A practically unconditionally gradient stable scheme for the N-component Cahn-Hilliard system. Physica A: Statistical Mechanics and its Applications.

LeVeque, R. (2004). Finite-volume methods for hyperbolic problems. Cambridge University Press. 
Novick-Cohen, A. and Segel, L. (1984). Nonlinear aspects of the Cahn-Hilliard equation. Physica D: Nonlinear Phenomena, 10(3):277-298.

Otto, F. and Giacomelli, L. (2005). New bounds for the Kuramoto-Sivashinsky equation. Communication on Pure and Applied Math., 58:0297-0318.

Podolny, A., Zaks, M., Rubinstein, B., Golovin, A., and Nepomnyashchy, A. (2005). Dynamics of domain walls governed by the convective Cahn-Hilliard equation. Physica D: Nonlinear Phenomena, 201(3):291-305.

Shen, J. and Yang, X. (2010). Numerical approximations of Allen-Cahn and Cahn-Hilliard equations. Discrete and Continuous Dynamical Systems, 28(4):1669-1691.

Shu, C.-W. (1997). Essentially non-oscillatory and weighted essentially non-oscillatory schemes for hyperbolic conservation laws. In ICASE Report, pages 325-432. Springer.

Shu, C.-W. and Osher, S. (1988). Efficient implementation of essentially non-oscillatory shock capturing schemes. Journal of Computational Physics, 77:439-471.

Tadmor, E. and Nessyahu, H. (1990). Non-oscillatory central differencing for hyperbolic conservation laws. Journal of Computational Physics, 87:408-463.

Ternat, F., Orellana, O., and Daripa, P. (2011). Two stable methods with numerical experiments for solving the backward heat equation. Applied Numerical Mathematics, 61(2):266284.

Watson, S. (2003). Crystal growth, coarsening and the convective Cahn-Hilliard equation. International series of Numerical Mathematics, 147:329-341.

Watson, S., Otto, F., Rubinstein, B., and Davis, S. (2003). Coarsening dynamics of the convective Cahn-Hilliard equation. Physical D, 178:127-148.

Wells, G. N., Kuhl, E., and Garikipati, K. (2006). A discontinuous Galerkin method for the Cahn-Hilliard equation. Journal of Computational Physics, 218(2):860-877.

Yong-Jung, K., Youngsoo, H., and Myers, T. (2008). On the numerical solution of a driven thin film equation. Journal of Computational Physics, 227:7246-7263.

Zachary, A. L., Malagoli, A., and Colella, P. (1994). A higher-order Godunov method for multidimensional ideal magnetohydrodynamics. SIAM Journal on Scientific Computing, $15(2): 263-284$.

Zhu, J., Chen, L.-Q., Shen, J., and Tikare, V. (1999). Coarsening kinetics from a variablemobility Cahn-Hilliard equation: Application of a semi-implicit fourier spectral method. Physical Review E, 60(4):3564. 